\title{
Childhood bladder and bowel dysfunction predicts irritable bowel syndrome phenotype in adult interstitial cystitis/bladder pain syndrome patients
}

\author{
R. Christopher Doiron, $M D^{\prime}$; Barry A. Kogan, $M D^{2}$; Victoria Tolls, BSC'; Karen Irvine-Bird; J. Curtis Nickel, $M D^{1}$
}

'Department of Urology, Queen's University, Kingston, ON, Canada; 2 Division of Urology, Albany Medical College, Albany, NY, United States

Cite as: Can Urol Assoc J 2017;11 (8):255-9. http://dx.doi.org/10.5489/cuaj.4251

\section{Abstract}

Introduction: Many clinicians have suggested that a history of bladder and bowel dysfunction (BBD) in childhood predisposes to the development of interstitial cystitis/bladder pain syndrome (IC/BPS) or irritable bowel syndrome (IBS) in adulthood. We hypothesized that BBD symptoms in childhood would predict the IBS-associated phenotype in adult IC/BPS patients.

Methods: Consecutive female patients $(n=190)$ with a diagnosis of IC/BPS were administered a modified form of a clinical BBD questionnaire (BBDQ) to capture childhood BBD-like symptoms, as well as Interstitial Cystitis Symptoms Index (ICSI), Interstitial Cystitis Problem Index (ICPI), Pelvic Pain and Urgency/ Frequency (PUF) questionnaires and UPOINT categorization. Patients were stratified to IBS-positive or IBS-negative according to clinical assessment of IBS-like symptoms.

Results: The 127 patients (67\%) identified with IBS-like symptoms recalled significantly higher BBDQ scores than the 63 patients $(33 \%)$ who were IBS-negative ( 2.8 vs. $2.3 ; p=0.05)$. The IBS-positive patients also reported a higher number of UPOINT domains than their non-IBS counterparts (3.8 vs. 2.9 ; $\mathrm{p}=0.0001)$, while their PUF total scores were significantly higher (13.6 vs. 12.3 ; $p=0.04)$. IBSpositive patients more often recalled that in childhood they did not have a daily bowel movement $(B M)(p=0.04)$ and had "to push for a BM" $^{\prime \prime}(p=0.009)$. In childhood, they "urinated only once or twice per day" ( $p=0.03)$ and recalled "painful urination" more than those without IBS $(p=0.03)$. There were no significant differences between the groups in answers to the other five questions of the BBDQ.

Conclusions: Our symptom recollection survey was able to predict the IBS phenotype of IC/BPS based on a childhood BBDQ. Further prospective studies are needed to further evaluate these novel findings.

\section{Introduction}

Interstitial cystitis/bladder pain syndrome (IC/BPS) patients represent a heterogeneous group of individuals, often with varied clinical phenotypes. ${ }^{1-3}$ Although lacking clear diagnostic criteria, the disease is characterized with pain in the bladder and pelvic floor and is associated with urinary frequency and urgency. The patho-etiology of IC/BPS has remained enigmatic, although several theories have been proposed. ${ }^{4-10}$ The demonstration from several clinicians ${ }^{2,11-15}$ of an association between childhood bladder and bowel dysfunction (BBD; previously called dysfunctional elimination syndrome or DES) and the development of lower urinary tract symptoms (LUTS) in adulthood supports a longitudinal process in some patients with overactive bladder $(\mathrm{OAB})$. The strong prevalence of irritable bowel syndrome (IBS) in patients with IC/BPS has been identified by many authors. ${ }^{16-20}$ The complex interplay of neurological, musculoskeletal, and immunological systems allows ample opportunity for early dysfunction of the bladder to be expressed in the adult as symptoms of IC/BPS, and because bowel dysfunction is clearly associated with bladder problems in children, this may well be found, especially in those adults with an IBS phenotype.

This longitudinal theory of progressive pathophysiology to account for some cases of adult IC/BPS has remained challenging to study, as no comprehensive transitional urology program appears to have prospective data to allow for such an analysis. Although fraught with limitations, an opportunity exists to evaluate patient recollections of BBD symptoms in childhood in an IC/BPS population. We explored the childhood history of BBD in female patients with IC/BPS and hypothesized that a history of childhood of BBD would predict the IBS-associated phenotype in female IC/BPS patients.

\section{Methods}

\section{Participants and study design}

Consecutive female patients with a diagnosis of IC/BPS from a single outpatient clinic who make up a large prospective 
clinical quality assurance database of IC/BPS patients were administered a modified BBD questionnaire (BBDQ). This patient sample has been described in previous publications. ${ }^{21}$ Briefly, the patient population consisted of English-speaking female patients who mostly would have met the IC/BPS diagnostic criteria as described in the IC Data Base Study. ${ }^{22}$

Patients were stratified to IBS-positive or IBS-negative according to clinical assessment of IBS-like symptoms using the Rome III criteria. ${ }^{23}$ The criteria requires recurrent abdominal pain or discomfort (uncomfortable sensation not described as pain) at least three days per month associated with two or more of: 1) improvement with defecation; 2 ) onset associated with change in frequency of stool; or 3) onset associated with change in form (appearance) of stool. ${ }^{23,24}$ The IBS-positive group was further substratified into those with a formal IBS diagnosis vs. those with IBS-like symptoms according to the Rome III criteria, but without a formal diagnosis of IBS. Further substratification among the IBS-positive group included IBS subtypes: IBS-constipation (IBS-C), IBS-diarrhea (IBS-D), and IBS-mixed (IBS-M). This study was done under ongoing institutional review board approval for continued quality assurance with all patient data de-identified before analysis.

\section{Measures}

All patients completed the Dysfunctional Voiding Symptom Score (DVSS) questionnaire, ${ }^{25}$ as well as the BBDQ, which was modified from the DVSS questionnaire to capture childhood BBD-like symptoms. The modified BBDQ addresses the following domains: constipation, urine holding, urinary continence, urinary urgency, obstruction, and dysuria. The modification asked the subject to recall if the symptoms were present in childhood, specifically between the ages of 8 and 10. Data regarding the patients' demographics, symptom duration, Interstitial Cystitis Symptoms Index (ICSI), Interstitial Cystitis Problem Index (ICPI) scores, ${ }^{26}$ Pelvic Pain and Urgency/ Frequency (PUF), UPOINT scoring, ${ }^{27}$ and the presence or absence of IBS (diagnosed and/or Rome III criteria) were collected through initial evaluations at the IC/ BPS outpatient clinic and has been previously reported. ${ }^{9}$

\section{Data analysis}

Statistical analysis was completed using Microsoft Excel 2010 Data Analysis package and the online Social Science Statistics software (http://www.socscistatistics.com). Age and relevant questionnaire scores (ICSI, UPOINT, ICPI, PUF, BBDQ) were analyzed as quantitative data using a two-tailed t-test assuming unequal variances. Categorical data including IBS subtype, diagnosed or undiagnosed, and DVSS and BBDQ categorical questions were analyzed using a two-tailed z-test for population proportions. All analysis was done used the statistical significance of 0.05 .

\section{Results}

Of the 190 patients assessed, 127 (67\%) were identified as having IBS-like symptoms, while the remaining 63 (33\%) did not. The IBS-positive patients did not recall having daily bowel movements (BMs) as children $(p=0.04)$ and remembered having to push during their BMs $(p=0.009)$ as children significantly more than their IBS-negative IC/BPS counterparts. Compared to those without IBS, they also recalled urinating only once or twice daily as children $(p=0.03)$, while their voiding in childhood was considered painful $(p=0.03)$. There were no significant differences in the remaining five questions of the childhood BBDQ (daytime/nighttime wetness, hold urinating by squatting and crossing legs, could not wait to void, and had to push to void). These results are shown in Table 1.

The IBS-positive patients' pushing during BMs and voiding persisted in adulthood significantly more than those with no IBS-like symptoms ( $p=0.0004$ and 0.003 , respectively; Table 1). The IC/BPS patients with IBS-like symptoms did have significantly more UPOINT domains reported (3.8 vs. 2.9; $\mathrm{p}=0.0001)$. Their total childhood BBDQ scores were also significantly higher than IC/BPS patients without IBS (2.8 vs. $2.3 ; p=0.05$ ). Furthermore, their PUF symptom score

\begin{tabular}{|c|c|c|c|}
\hline $\begin{array}{l}\text { Between the ages of } 8 \text { and } \\
10 \text {, average (SD) }\end{array}$ & $\begin{array}{l}\text { IBS-yes } \\
\text { ( } n=127)\end{array}$ & $\begin{array}{l}\text { IBS-no } \\
(n=63)\end{array}$ & $\mathbf{p}$ \\
\hline Wet clothes daytime & $22(17.3)$ & $8(12.1)$ & 0.4 \\
\hline Wet clothes nighttime & $27(21.3)$ & $7(11.1)$ & 0.08 \\
\hline No daily BM & $62(48.8)$ & $21(33.3)$ & 0.04 \\
\hline Push during BM & $55(43.3)$ & $15(23.8)$ & 0.009 \\
\hline Urinate once or twice daily & $31(24.4)$ & $25(39.7)$ & 0.03 \\
\hline $\begin{array}{l}\text { Hold urine by crossing legs } \\
\text { or squatting }\end{array}$ & 76 (59.8) & $44(69.8)$ & 0.2 \\
\hline Could not wait to void & $36(28.3)$ & $13(20.6)$ & 0.3 \\
\hline Had to push to void & $21(16.5)$ & $5(7.9)$ & 0.1 \\
\hline Painful urination & $26(20.5)$ & $5(7.9)$ & 0.03 \\
\hline $\begin{array}{l}\text { Over the past month, } \\
\text { average (SD) }\end{array}$ & $\begin{array}{l}\text { IBS-yes } \\
\text { (n=127) }\end{array}$ & $\begin{array}{l}\text { IBS-no } \\
\text { (n=63) }\end{array}$ & $\mathbf{p}$ \\
\hline Wet clothes daytime & $64(50.4)$ & $24(38.1)$ & 0.1 \\
\hline Wet clothes nighttime & $22(17.3)$ & $5(7.9)$ & 0.08 \\
\hline No daily BM & $71(55.9)$ & $28(44.4)$ & 0.1 \\
\hline Push during BM & $77(60.6)$ & $21(33.3)$ & 0.0004 \\
\hline Urinate once or twice daily & $5(3.9)$ & $1(1.6)$ & 0.4 \\
\hline $\begin{array}{l}\text { Hold urine by crossing legs } \\
\text { or squatting }\end{array}$ & $46(36.2)$ & $23(36.5)$ & 1.0 \\
\hline Could not wait to void & $84(66.1)$ & $38(60.3)$ & 0.4 \\
\hline Painful urination & $80(63.0)$ & $32(50.8)$ & 0.1 \\
\hline Had to push to void & $59(46.5)$ & $15(23.8)$ & 0.003 \\
\hline
\end{tabular}

BBDO: bladder and bowel dysfunction questionnaire; BM: bowel movements; IBS: irritable bowel syndrome; IC/BPS: interstitial cystitis/bladder pain syndrome; SD: standard deviation. 
was significantly increased ( 13.6 vs. $12.3 ; \mathrm{p}=0.04$ ); however, their ICSI, ICPI, and total PUF scores, although numerically increased, were not significantly different (Table 2).

A stratified analysis looking at those diagnosed with IBS vs. those found to have IBS-like symptoms according to Rome III criteria, but without a formal IBS diagnosis, did not show any significant differences in the BBDQ (data not shown). A further stratified analysis compared subtypes of IBS, including IBS-C, IBS-D, and IBS-M (Table 3). The constipated subtype pushes significantly more during BMs as adults than both the diarrhea subtype and mixed subtype $(p=0.0001$ and 0.02 , respectively) while the IBS-M subtype pushes significantly more than the IBS-D group ( $p=0.004)$. The IBS-C group also noted significantly more "wet clothes" as an adult than the diarrhea group ( $p=0.001)$, while the IBS-M subtype was also significantly wetter than the diarrhea group $(p=0.03)$. The only significant differences noted in their childhood symptoms were that the IBS-C group pushed more during BMs than the IBS-M group ( $p=0.04)$, but not statistically significantly more than the IBS-D group ( $p=0.09$ ). The constipated subtype also noted a higher incidence of only urinating once or twice daily than the mixed subtype $(p=0.04)$.

\section{Discussion}

We report childhood BBD symptoms in a cohort of female IC/BPS patients evaluated at a single outpatient clinic. IC/ BPS patients with IBS-like symptoms report significantly more BBD symptoms (significantly higher total modified childhood BBDQ scores) and more adult UPOINT domains than their counterparts without IBS-like symptoms. During childhood, those with adult IBS reported significant differences than those without IBS in that they did not have daily BMs, had to push during BMs, voided only once or twice daily, and had painful urination. Although their PUF symptom score was found to be statistically significantly increased as well, this does not likely represent a clinically significant difference.

Several previous studies have reported an association between childhood BBD and adult voiding symptoms. Fitzgerald et $\mathrm{al}^{13}$ administered self-reported questionnaires to a population-based cohort of healthy women and found that several childhood urinary symptoms were predictive of $\mathrm{OAB}$ symptoms in adult women. Moore et $\mathrm{al}^{15}$ reported that in 1000 consecutive patients undergoing urodynamic studies, one-third of women and two-thirds of men found to have idiopathic detrusor instability had a history of troublesome enuresis in childhood. Bower et al ${ }^{14}$ reported that 191 consecutive patients presenting to their urogynecology clinic had higher childhood BBDQ scores than 252 normal women.

These studies, although suggestive of a longitudinal process of bladder and pelvic floor dysfunction, are not directly applicable to the IC/BPS population, as the patients studied were at times undifferentiated and lacked clear diagnoses. Peters et $\mathrm{l}^{11}$ found significantly increased rates of recurrent urinary tract infections (UTIs) and antibiotic usage in childhood of a cohort of IC/BPS patients $(n=215)$ compared to a healthy control population. Furthermore, their IC/BPS patients reported significantly higher rates of urinary urgency, trouble initiating stream, constipation, and painful defecation during childhood compared with the control group.

Our current study is the first of its kind to stratify IC/ BPS patients into those with IBS-like symptoms and those without when inquiring about childhood symptoms of BBD. We had hypothesized that the IBS-associated phenotype of IC/BPS patients would more strongly exhibit childhood BBD symptoms and our findings indeed support this. Furthermore, our substratification of IBS-positive patients suggest that the IBS-C group may represent patients who suffered more significantly in childhood. It is furthermore interesting to note that the IBS-C group had numerically higher rates of incontinence in adulthood compared to both the IBS-D and IBS-M groups - albeit the difference was only statistically significant compared to the IBS-D group. This finding may further support that worse childhood symptoms, particularly pushing during BMs and infrequent urination, may manifest in more significant voiding dysfunction in adulthood.

The limitations of this current study are obvious. The exercise of recalling childhood symptoms in a group of IC/ BPS patients is undoubtedly wrought with bias. It is however, the only childhood data we have in this population, as no prospective studies of pediatric patients with BBD exist in the literature. In an attempt to mitigate this recall bias, we did specify in the questionnaire for patients to recall symptoms specifically between the ages of 8 and 10 . We felt this was an age that would allow for reasonable recollection, but at

\begin{tabular}{|c|c|c|c|c|c|c|c|c|c|}
\hline & $\mathbf{n}$ & Age & $\begin{array}{l}\text { BBD child } \\
\text { total }\end{array}$ & $\begin{array}{c}\text { UPOINT } \\
\text { total }\end{array}$ & ICSI total & ICPI total & $\begin{array}{l}\text { Sx score } \\
\text { total PUF }\end{array}$ & $\begin{array}{l}\text { Bother score } \\
\text { total PUF }\end{array}$ & PUF total \\
\hline IBS-yes & 127 & $45.8(16.8)$ & 2.8 (1.9) & $3.8(1.4)$ & $13.7(4.3)$ & $11.6(3.7)$ & $13.6(4.8)$ & $7.0(2.9)$ & $20.6(7.4)$ \\
\hline IBS-no & 63 & $48.9(18.7)$ & $2.3(1.6)$ & $2.9(1.3)$ & $12.9(3.9)$ & $11.1(3.4)$ & $12.3(3.6)$ & $6.7(2.5)$ & $18.9(5.5)$ \\
\hline $\mathrm{p}$ & & 0.3 & 0.05 & 0.0001 & 0.2 & 0.4 & 0.04 & 0.4 & 0.09 \\
\hline
\end{tabular}




\begin{tabular}{|c|c|c|c|c|c|c|}
\hline IBS types & IBS-M & IBS-C & IBS-D & IBS-M vs. IBS-C & IBS-M vs. IBS-D & IBS-C vs. IBS-D \\
\hline $\mathrm{n}$ & 32 & 43 & 33 & $\mathbf{p}$ & $\mathbf{p}$ & $\mathbf{p}$ \\
\hline \multicolumn{7}{|c|}{ Between the ages of 8 and 10, average (SD) } \\
\hline Wet clothes daytime & $4(12.5)$ & $8(18.6)$ & $7(21.2)$ & 0.5 & 0.3 & 0.9 \\
\hline Wet clothes nighttime & $8(25.0)$ & $7(16.3)$ & $8(24.2)$ & 0.4 & 0.9 & 0.4 \\
\hline No daily BM & $15(46.9)$ & $25(58.1)$ & $13(39.4)$ & 0.4 & 0.5 & 0.1 \\
\hline Push during BM & 10 (31.3) & $24(55.8)$ & $12(36.4)$ & 0.03 & 0.7 & 0.09 \\
\hline Urinate once or twice daily & $4(12.5)$ & $14(32.6)$ & $6(18.2)$ & 0.04 & 0.5 & 0.2 \\
\hline $\begin{array}{l}\text { Hold urine by crossing } \\
\text { legs or squatting }\end{array}$ & $21(65.6)$ & $25(58.1)$ & $18(54.5)$ & 0.5 & 0.4 & 0.8 \\
\hline Could not wait to void & $13(40.6)$ & $13(30.2)$ & $8(24.2)$ & 0.3 & 0.2 & 0.6 \\
\hline Had to push to void & 7 (21.9) & $6(14.0)$ & $5(15.2)$ & 0.4 & 0.5 & 0.9 \\
\hline Painful urination & $9(28.1)$ & $8(18.6)$ & $8(24.2)$ & 0.3 & 0.7 & 0.6 \\
\hline \multicolumn{7}{|c|}{ Over the past month, average (SD) } \\
\hline Wet clothes daytime & $18(56.3)$ & $29(67.4)$ & $10(30.3)$ & 0.3 & 0.03 & 0.001 \\
\hline Wet clothes nighttime & $5(15.6)$ & $7(16.3)$ & $6(18.2)$ & 0.9 & 0.9 & 0.8 \\
\hline No daily BM & $20(62.5)$ & $27(62.8)$ & $13(39.4)$ & 1.0 & 0.06 & 0.04 \\
\hline Push during BM & $20(62.5)$ & $37(86.0)$ & $9(27.3)$ & 0.02 & 0.004 & 0.0 \\
\hline Urinate once or twice daily & $2(6.3)$ & $3(7.0)$ & $0(0.0)$ & 0.9 & 0.1 & 0.1 \\
\hline $\begin{array}{l}\text { Hold urine by crossing } \\
\text { legs or squatting }\end{array}$ & $11(34.4)$ & $18(41.9)$ & $13(39.4)$ & 0.5 & 0.7 & 0.8 \\
\hline Could not wait to void & $21(65.6)$ & $29(67.4)$ & $24(72.7)$ & 0.9 & 0.5 & 0.6 \\
\hline Had to push to void & $23(71.9)$ & $30(69.8)$ & $21(63.6)$ & 0.8 & 0.5 & 0.6 \\
\hline Painful urination & $13(40.6)$ & $20(46.5)$ & $16(48.5)$ & 0.6 & 0.5 & 0.9 \\
\hline
\end{tabular}

BBDQ: bladder and bowel dysfunction questionnaire; BM: bowel movements; IBS: irritable bowel syndrome; IC/BPS: interstitial cystitis/bladder pain syndrome; SD: standard deviation.

which the prevalence of BBD would still be high enough to include those who might have suffered.

Furthermore, our current dataset lacks any information about the state of the patients' disease at the time of questionnaire administration, which could further bias their recollection and was not controlled for. The questionnaire used, although not validated, was modified from a validated questionnaire ${ }^{25}$ used in the pediatric population. No questionnaires have been fully validated to recall childhood symptoms of BBD and as a result, the best data we can come up with is our patients recalling symptoms as children. Our study is further limited by a lack of control population.

This study provides further insight into a longitudinal theory of BBD and provides new insight into the IBS-associated phenotype of IC/BPS patients. It again highlights a glaring lack of literature studying the longitudinal progression theory of adult IC/BPS developing from symptomatic children. As comprehensive transitional pediatric to adult urology programs develop, one objective should be a prospective database that will answer these type of questions.

\section{Conclusion}

IBS-positive IC/BPS patients report higher total BBDQ scores as children and more reported UPOINT domains as adults than IC/BPS patients without IBS. The IBS-C subtype recall suffering worse in childhood and manifest more significant symptoms in adulthood. Prospective studies in pediatric patients with elimination disorders are sorely needed to confirm the longitudinal theory of IC/BPS progression.

Competing interests: Dr. Nickel has been a consultant for Astellas, Auxillium, Eli Lilly, Farr Labs, Ferring, GSK, Pfizer, Taris Biomedical, Tribute, and Trillium Therapeutics; a speaker for Astellas and Eli Lilly; and has participated in clinical trials supported by GSK, Johnson \& Johnson, Pfizer, and Taris Biomedical. The remaining authors report no competing personal or financial interests.

This paper has been peer-reviewed.

\section{References}

1. Nickel JC. Words of wisdom. Re: clinical phenotyping in chronic prostatitis/chronic pelvic pain syndrome and interstitial cystitis: A management strategy for urological chronic pelvic pain syndromes. Eur Urol 2009;56:881. https://doi.org/10.1016/i.eururo.2009.08.005

2. Shoskes DA, Nickel JC, Rackley RR, et al. Clinical phenotyping in chronic prostatitis/chronic pelvic pain syndrome and interstitial cystitis: A management strategy for urological chronic pelvic pain syndromes. Prostate Cancer Prostatic Dis 2009;12:177-83. https://doi.org/10.1038/pcan.2008.42

3. Nickel IC, Tripp DA. International Interstitial Cystitis Study Group. Clinical and psychological parameters associated with pain pattern phenotypes in women with interstitial cystitis/bladder pain syndrome. J Urol 2015;193:138-44. https://doi.org/10.1016/i.juro.2014.07.108

4. Parsons (LL, Lilly JD, Stein P. Epithelial dysfunction in non-bacterial cystitis (interstitial cystitis). J Urol 1991;145:732-5. https://doi.org/10.1016/S0022-5347(17)38437-9

5. Argade $S$, Shaw $T$, Su $Y$, et al. Tamm-Horsfall protein-associated nucleotides in patients with interstitial cystitis. BJU Int 2013;111:811-9. https://doi.org/10.1111/ן.1464-410X.2012.11691.x

6. Peeker R, Enerback L, Fall $M$, et al. Recruitment, distribution, and phenotypes of mast cells in interstitial cysitis. J Urol 2000;163:1009-15. https://doi.org/10.1016/S0022-5347(05)67873-1 
7. Warren JW, Brown V, Jacobs $S$, et al. Urinary tract infection and inflammation at onset of interstitial cystitis/painful bladder syndrome. Urology 2008;71:1085-90. https://doi.org/10.1016/j.urology.2007.12.091

8. Warren JW, Jackson TL, Langenberg $\mathrm{P}$, et al. Prevalence of interstitial cystitis in first-degree relatives of patients with interstitial cystitis. Urology 2004;63:17-21. https://doi.org/10.1016/j.urology.2003.08.027

9. MacDermott JP, Miller CH, Levy N, et al. Cellular immunity in interstitial cystitis. J Urol 1991;145:274-8. https://doi.org/10.1016/S0022-5347(17)38313-1

10. Warren JW, van de Merwe JP, Nickel JC. Interstitial cystitis/bladder pain syndrome and non-bladder syndromes: Facts and hypotheses. Urology 2011;78:727-32. https://doi.org/10.1016/j.urology.2011.06.014

11. Peters KM, Killinger KA, Ibrahim IA. Childhood symptoms and events in women with interstitial cystitis/ painful bladder syndrome. J Urol 2009;73:258-62. htrps://doi.org/10.1016/i.urology.2008.09.014

12. Minassian VA, Lovatsis $D$, Pascali $D$, et al. Effect of childhood dysfunctional voiding on urinary incontinence in adult women. Obstet Gynecol 2006;107:1247-51. https://doi.org/10.1097/01. AOG.0000190222.12436.38

13. Fitzgerald MP, Thom DH, Wassel-Fyr C, et al. Childhood urinary symptoms predict adult overactive bladder symptoms. J Urol 2006;175:989-93. https://doi.org/10.1016/S0022-5347(05)00416-7

14. Bower WF, Yip SK, Yeung CK. Dysfunctional elimination symptoms in childhood and adulthood. I Urol 2005;174:1623-27. https://doi.org/10.1097/01.ju.0000176599.91836.12

15. Moore KH, Richmond DH, Parys BT. Sex distribution of adult idiopathic detrusor instability in relation to childhood bedwetting. Br J Urol 1991;68:479-82. https://doi.org/10.1111/j.1464-410X.1991.tb15389.x

16. Nickel JC, Tripp DA, Pontari M, et al. Interstitial cystitis/painful bladder syndrome and associated medical conditions with an emphasis on irritable bowel syndrome, fibromyalgia, and chronic fatigue syndrome. J Urol 2010;184:1358-63. https://doi.org/10.1016/i.juro.2010.06.005

17. Reed BD, Harlow SD, Sen A, et al. Relationship between vulvodynia and chronic comorbid pain conditions. Obstet Gynecol 2012;120:145-51. https://doi.org/10.1097/AOG.0b013e31825957cf

18. Warren JW, Howard FM, Cross RK, et al. Antecedent non-bladder syndromes in case-control study of interstitial cystitis/painful bladder syndrome. Urology 2009;73:52-7. https://doi.org/10.1016/i. urology.2008.06.031
19. Rodriguez MA, Afari N, Buchwald DS, National Institute of Diabetes and Digestive and Kidney Diseases Working Group on Urological Chronic Pelvic Pain: Evidence for overlap between urological and nonurological unexplained clinical conditions. J Urol 2009;182:2123-31. https://doi.org/10.1016/i. juro.2009.07.036

20. Novi JM, Jeronis $S$, Srinivas $S$, et al. Risk of irritable bowel syndrome and depression in women with interstitial cystitis: A case-control study. J Urol 2005;174:937-40. https://doi.org/10.1097/01. ju.0000169258.31345.5d

21. Nickel JC, Tripp DA, Pontari $M$, et al. Psychosocial phenotyping in women with interstitial cystitis/painful bladder syndrome: A case control study. J Urol 2010;183:167-72. https://doi.org/10.1016/i. juro.2009.08.133

22. Hanno PM, Landis JR, Matthews-Cook Y, et al. Diagnosis of interstitial cystitis revisited: Lessons learned from the National Institutes of Health, Interstitial Cystitis Data Base Study. J Urol 1999;161:553-7. https://doi.org/10.1016/S0022-5347(01)61948-7

23. Drossman DA, Dumitrascu DL. Rome III: New standard for functional gastrointestinal disorders. J Gastrointestin Liver Dis 2006; 15:237-41.

24. Longstreth GF, Thompson WG, Chey WD, et al. Functional bowel disorders. Gastroenterology 2006;130:1480-91. https://doi.org/10.1053/i.gastro.2005.11.06

25. Farhat W, Bagli DJ, Capolicchio $G$, et al. The dysfunctional voiding scoring system: Quantitative standardization of dysfunctional voiding symptoms in children. J Urol 2000;164:101 1-5. https://doi.org/10.1016/ S0022-5347(05)67239-4

26. O'Leary MP, Sant GR, Fowler FJ Jr, et al. The interstitial cystitis symptom index and problem index. Urology 1997;49:58-63. https://doi.org/10.1016/S0090-4295(99)80333-1

27. Nickel JC, Shoskes D, Irvine-Bird K. Clinical phenotyping of women with interstitial cystitis/painful bladder syndrome (IC/PBS): A key to classification and potentially improved management. J Urol 2009;182:155160. https://doi.org/10.1016/i.juro.2009.02.122

Correspondence: Dr. J. Curtis Nickel, Queen's University, Kingston, ON, Canada; icn@queensu.ca

\section{NEW FEATURE!}

\section{CUAJ is now offering its readers the opportunity to claim Section 3 Self-Assessment credits of the Maintenance of Certification (MOC) Program!}

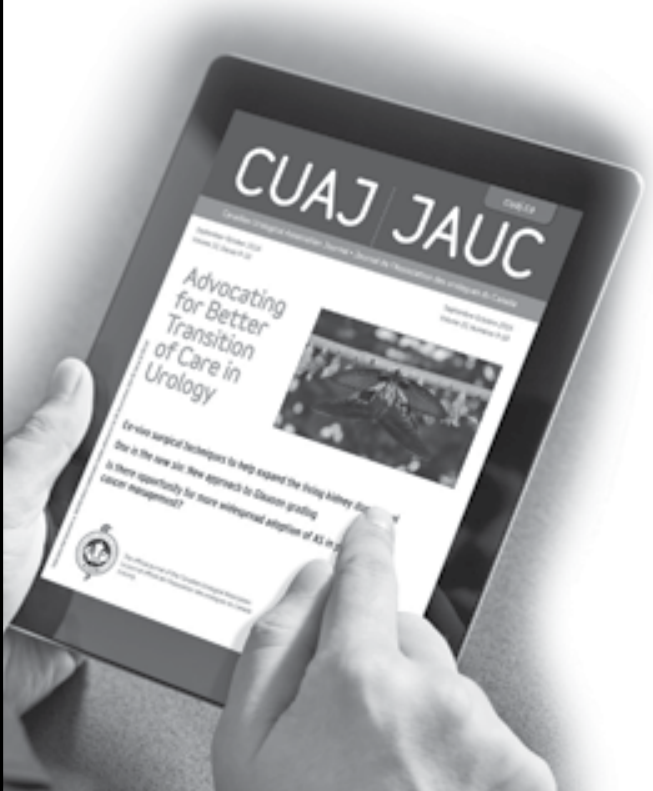

Every second issue (February, April, June, August, October, and December), a specific paper will be accredited by the Canadian Urological Association (CUA).

\section{CLAIM YOUR CREDITS IN 3 EASY STEPS:}

(1) Go to www.cuaj.ca, read the accredited paper, and answer the three multiple choice questions associated with it.

2 Enter your name and email to receive a certificate of participation from CUA.

3 Log the self-learning activity and record learning/outcomes in your Royal College MAINPORT account.

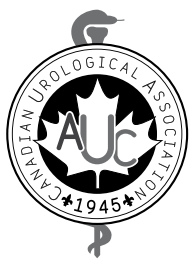

